\title{
Empirical Analysis of an Online Algorithm for Multiple Trading Problems
}

\author{
Esther Mohr ${ }^{1)}$ \\ Günter Schmidt ${ }^{1+2)}$ \\ 1) Saarland University \\ 2) University of Liechtenstein \\ $\underline{\text { em@itm.uni-sb.de }}$ \\ gs@itm.uni-sb.de
}

\begin{abstract}
If we trade in financial markets we are interested in buying at low and selling at high prices. We suggest an active trading algorithm which tries to solve this type of problem. The algorithm is based on reservation prices. The effectiveness of the algorithm is analyzed from a worst case and an average case point of view. We want to give an answer to the questions if the suggested active trading algorithm shows a superior behaviour to buy-andhold policies. We also calculate the average competitive performance of our algorithm using simulation on historical data.
\end{abstract}

Keywords: online algorithms, average case analysis, stock trading, trading rules, performance analysis, competitive analysis, trading problem, empirical analysis

\section{Introduction}

Many major stock markets are electronic market places where trading is be carried out automatically. Trading policies which have the potential to operate without human interaction are of great importance in electronic stock markets. Very often such policies are based on data from technical analysis [She02, RL99, RS03]. Many researchers have also studied trading policies from the perspective of artificial intelligence, software agents and neural networks [CE08, FRY04, SR05].

In order to carry out trading policies automatically they have to be converted into trading algorithms. Before a trading algorithm is applied one might be interested in its performance. The performance analysis of trading algorithms can basically be carried by three different approaches. One is Bayesian analysis where a given probability distribution for asset prices is a basic assumption. Another one is assuming uncertainty about asset prices and analysing the trading algorithm under worst case outcomes; this approach is called competitive analysis. The third one is a heuristic approach where trading algorithms are 
designed and the analysis is done on historic data by simulation runs. In this paper we apply the second and the third approach in combination. We consider a multiple trade problem and analyse an appropriate trading algorithm from a worst case point of view. Moreover we evaluate its average case performance empirically and compare it to other trading algorithms.

The reminder of this paper is organized as follows. In the next section we formulate the problem and perform a worst case competitive analysis of the proposed trading algorithm. In Section 3 different trading policies for the multiple trade problem are introduced. Section 4 gives a literature overview on heuristic trading rules for multiple trading problems. In Section 5 detailed experimental findings from our simulation runs are presented. We finish with some conclusions in the last section.

\section{Problem Formulation}

If we trade in financial markets we are interested in buying at low prices and selling at high prices. Let us consider the single trade and the multiple trade problem. In a single trade problem we search for the minimum price $m$ and the maximum price $M$ in a time series of prices for a single asset. At best we buy at price $m$ and sell later at price $M$. In a multiple trade problem we trade assets sequentially in a row, e.g. we buy some asset $u$ today and sell it later in the future. After selling asset $u$ we buy some other asset $v$ and sell it later again; after selling $v$ we can buy $w$ which we sell again, etc. If we buy and sell (trade) assets $k$ times we call the problem $k$-trade problem with $k \geq 1$.

As we do not know future prices the decisions to be taken are subject to uncertainty. How to handle uncertainty for trading problems is discussed in [YFKT01]. In [Yan98] and [YFKT92] online algorithms are applied to a search problem. Here a trader owns some asset at time $t=0$ and obtains a price quotation $m \leq p(t) \leq M$ at points of time $t=1,2, \ldots, T$. The trader must decide at every time $t$ whether or not to accept this price for a sell. Once some price $p(t)$ is accepted trading is closed and the trader's payoff is calculated. The horizon $T$ and the possible minimum and maximum prices $m$ and $M$ are known to the trader. If the trader did not accept a price at the first $T-1$ points of time he must be prepared to accept some minimum price $m$ at time $T$. The problem is solved by an online algorithm.

An algorithm ON computes online if for each $j=1, \ldots, n-1$, it computes an output for $j$ before the input for $j+1$ is given. An algorithm computes offline if it computes a feasible output given the entire input sequence $j=1, \ldots, n-1$. We denote an optimal offline algorithm by OPT. An online algorithm ON is $c$-competitive if for any input $I$ 


$$
\mathrm{ON}(I) \geq 1 / c * \mathrm{OPT}(I)
$$

The competitive ratio is a worst-case performance measure. In other words, any $c$ competitive online algorithm is guaranteed a value of at least the fraction $1 / c$ of the optimal offline value OPT $(I)$, no matter how unfortunate or uncertain the future will be. When we have a maximization problem $c \geq 1$, i.e. the smaller $c$ the more effective is ON. For the search problem the policy (trading rule) [Yan98]

accept the first price greater or equal to reservation price $p^{*}=\sqrt{\left(M^{*} m\right)}$

has a competitive ratio $c_{s}=\sqrt{\frac{M}{m}}$ where $M$ and $m$ are upper and lower bounds of prices $p(t)$ with $p(t)$ from $[m, M]$.

This result can be transferred to $k$-trade problems if we modify the policy to

buy the asset at the first price smaller or equal and sell the asset at the first price greater or equal to reservation price $p^{*}=\sqrt{\left(M^{*} m\right)}$

In the single trade problem we have to carry out the search twice. In the worst case we get a competitive ratio of $c_{s}$ for buying and the same competitive ratio of $c_{s}$ for selling resulting in an overall competitive ratio for the single trade problem of $c_{t}=c_{s} c_{s}=M / m$. In general we get for the $k$-trade problem a competitive ratio of $c_{t}(k)=\prod_{i=1, \ldots, k}(M(i) / m(i))$. If $m$ and $M$ are constant for all trades $c_{t}(k)=(M / m)^{k}$. The ratio $c_{t}$ can be interpreted as the rate of return we can achieve by buying and selling assets.

The bound is tight for arbitrary $k$. Let us assume for each of $k$ trades we have to consider the time series $\left(M,(M m)^{1 / 2}, m, m,(M m)^{1 / 2}, M\right)$. OPT always buys at price $m$ and sells at price $M$ resulting in a return rate of $M / m$; ON buys at price $(M m)^{1 / 2}$ and sells at price $(\mathrm{Mm})^{1 / 2}$ resulting in a return rate of 1 , i.e. OPT/ON $=M / m=c$. If we have $k$ trades OPT will have a return of $(M / m)^{k}$ and $\mathrm{ON}$ of $1^{k}$, i.e. $\mathrm{OPT}(k) / \mathrm{ON}(k)=(M / m)^{k}=c(k)$.

In the following we apply the above modified reservation price policy to multiple trade problems. 


\section{Multiple Trade Problem}

In a multiple trade problem we have to choose points of time for selling current assets and buying new assets over a known time horizon. The horizon consists of several trading periods $i$ of different types $p$; each trading period consists of a constant number of $h$ days. We differ between $p=1,2, \ldots, 6$ types of periods with length $h$ from $\{7,14,28,91,182,364\}$ days e.g. period type $p=6$ has length $h=364$ days; periods of type $p$ are numbered with $i=1, \ldots, n(p)$. There is a fixed length $h$ for each period type $p$, e.g. period length $h=7$ corresponds to period type $p=1$, period length $h=14$ corresponds to period type $p=2$, etc. For a time horizon of one year, for period type $p=1$ we get $n(1)=52$ periods of length $h=7$, for type $p=2$ we get $n(2)=26$ periods of length $h=14$, etc.

We may choose between three trading policies. Two elementary ones are Buy-andHold $(\mathrm{B}+\mathrm{H})$, a passive policy, and Market Timing (MT), an active policy. The third one is a random (Rand) policy. As a benchmark we use an optimal offline algorithm called Market (MA). We assume that for each period $i$ there is an estimate of the maximum price $M(i)$ and the minimum price $m(i)$. Within each period $i=1, \ldots, n(p)$ we have to buy and sell an asset at least once. The annualized return rate $R(x)$, with $x$ from $\{\mathrm{MT}$, Rand, $\mathrm{B}+\mathrm{H}, \mathrm{MA}\}$ is the performance measure used. At any point of time of the horizon the policy either holds an asset or an overnight deposit.

In order to describe the different policies we define a holding period with respect to MT. A holding period is the number of days $h$ between the purchase of asset $j$ and the purchase of another asset $j^{\prime}\left(j^{\prime} \neq j\right)$ by MT. Holding periods are determined by either reservation prices $R P_{j}(t)$ which give a trading signal or when the last day $\mathrm{T}$ of the period is reached.

\section{MARKET TIMING (MT)}

MT calculates reservation prices $R P_{j}(t)$ for each day $t$ for each asset $j$. At each day $t$, MT must decide whether to sell asset $j$ or to hold it another day considering the reservation prices. Each period $i$, the first offered price $p_{j}(t)$ of asset $j$ with $p_{j}(t) \geq R P_{j}(t)$ is accepted by MT and asset $j$ is sold. The asset $j^{*}$, which is bought by MT is called MTasset. MT chooses the MTasset $j^{*}$ if $R P_{j^{*}}(t)-p_{j^{*}}(t)=\max \left\{R P_{j}(t)-p_{j}(t) \mid j=1, \ldots, m\right\}$ and $p_{j^{*}}(t)<R P_{j^{*}}(t)$. If there was no trading signal in a period related to reservation prices then trading is done on the last day $T$ of a period. In this case MT must sell asset $j$ and invest in asset $j^{\prime}$ at $T$. The holding period showing buying (Buy) and selling (Sell) points of time and an interval with overnight deposit (OD) for MT is shown in Figure 1. 


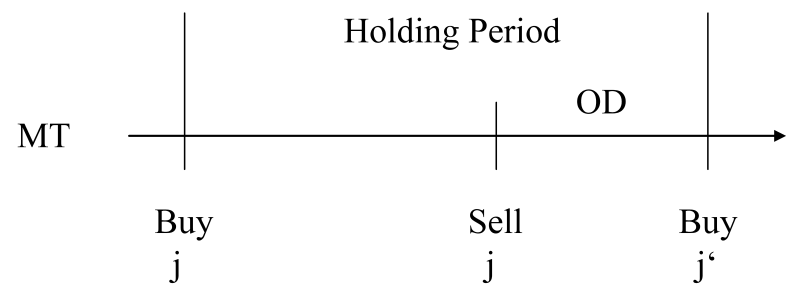

Figure 1: Holding period for MT

\section{RANDOM (Rand)}

Rand will buy and sell at randomly chosen prices $p_{j}(t)$ within the holding period of MT (cf. Figure 2).

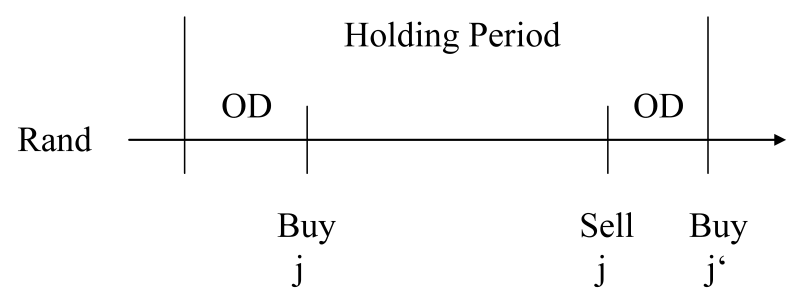

Figure 2: Trading Possibilities for Rand

\section{BUY AND HOLD $(\mathrm{B}+\mathrm{H})$}

$\mathrm{B}+\mathrm{H}$ will buy at the first day $t$ of the period and sell at the last day $T$ of the period.

\section{MARKET (MA)}

To evaluate the performance of these three policies empirically we use as a benchmark the optimal offline policy. It is assumed that MA knows all prices $p_{j}(t)$ of a period including also these which were not presented to MT if there were any. In each period $i$ MA will buy at the minimum price $p_{\min } \geq m(i)$ and sell at the maximum possible price $p_{\max } \leq M(i)$ within the holding period of MT (cf. Figure 3).

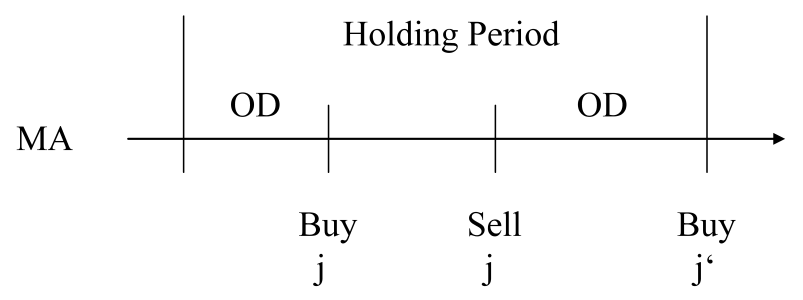

Figure 3: Trading possibilities for MA

The performance of the investment policies is evaluated empirically. Clearly, all policies cannot beat the benchmark policy MA. The policies are tested using historical 
XETRA DAX data for a consecutive 364-day period from 2007.01.01 to 2007.12.31 (see Section 5).

\section{Heuristic Trading Policies}

We give a brief overview on the experimental analysis on heuristic trading policies for multiple trade problems. The policies discussed in [She02] and [CE08] outperformed the buyand-hold policy on the selected data set.

In [She02] simple market-timing heuristics are investigated that outperform the buyand-hold policy with data from 1970 to 2000. Trading Signals are generated by the value of the short spread between the Earning/Price (E/P) ratio of the S\&P500 index and selected interest rates. The earnings are forecasted for the coming period and divided by the actual asset price. Trading policies either invest in the S\&P500 index or in treasury bills over a period of one month depending on predefined thresholds. If the spread is above some threshold level, it is invested in the market index for the next month and if the spread is below it, the portfolio is liquidated at the end of the month and the money is invested in 30-day treasury-bills for the next month. At the end of each month spreads are considered again. As a benchmark portfolio values are compared with these of S\&P500 index buy-and-hold from 1970 to 2000. Results show that all trading policies outperform the S\&P500 index generating higher mean returns. In particular, the policy based on the spread between the E/P ratio and a short-term interest rate beats the market index even when transaction costs are incorporated.

[CE08] explore the profitability of stock trading by using a neural network (NN) to assist the trading decisions which are based on two technical indicators, the volume adjusted moving average (VAMA) and the ease of movement (EMV) indicator. VAMA is a moving average, where prices are replaced by volume. EMV illustrates the relationship between the rates of price and volume change of an asset. Trading is simulated over a time horizon of 1508 days from January 1998 to December 2003. At each time only one asset of the S\&P500 index is in the portfolio. Different types of period lengths are investigated: 1 week (5-days), 4 weeks (21-days) and 13 weeks (55-days). Trading signals are generated by VAMA and EMV with and without NN support. Transaction costs are not considered. The VAMA rule buys if the price of the asset is smaller than the VAMA and sells if the price is greater. The EMV trading rule buys when the smoothing value of EMV crosses above zero from below and sells when the smoothing value of EMV crosses below zero from above. Trading rules might not be executed depending on the results of the NN which predict the next day's VAMA and 
EMV. Benchmarks are VAMA, EMV, a single moving average (SMA) and buy-and-hold. Different combinations of trading policies are tested: VAMA + NN, VAMA + NN + Filter, $\mathrm{VAMA}+\mathrm{NN}+\mathrm{SMA}$, and EMV + NN + VAMA. For the combined cases, trading signals must match for all components (for more details see Tables 6 and 7 in [CE08]). Results show that trading with NN support is helpful to generate better trading decisions. The EMV + NN + VAMA policy outperforms all benchmarks.

[RL99] examine variable length moving average (VMA) trading rules in ten emerging equity markets in Latin America and Asia from January 1982 to April 1995. The average returns incorporating trading costs for each rule and country are compared to buy-and-hold the S\&P500 and Nikkei225 indices. Trading signals are generated by two different types of moving average returns (MAR), called short MAR and long MAR. The short MAR is calculated over period lengths of 1, 2, and 5 days, the long MAR over 50, 150, and 200 days. Six VMA trading policies generate buying signals when the short MAR exceeds the long MAR and selling signals when the long MAR exceeds the short MAR. They were tested over a time horizon from January 1982 to April 1995. Results show that VMA trading rules applied to emerging markets do not beat the buy-and-hold alternative.

In [RS03] two trading policies are suggested which are applied in [FRY04] to stock trading agents in the context of the Penn-Lehman Automated Trading simulator. The first agent represents a market-making policy exploiting market volatility without predicting the direction of the stock price movement. The second agent uses technical analysis. Both agents trade over 15 days from February $24^{\text {th }}$ to March $18^{\text {th }} 2003$ the Microsoft Corp. (MSFT) asset. The market-making policy fixes a selling price $x$ and a buying price $y$ for MSFT. When prices go beyond $x$ a sell order is placed when prices drop on $y$ a buy order. The reverse policy sells when prices go upwards and buys when prices go downwards. The experimental analysis is designed as a tournament with three rounds, each lasting one week. Both policies survived the first round; the market-making policy did not survive the second round. The reverse policy won the tournament but without achieving any profit. Here buy-and-hold was not simulated.

In [SR05] the potential of combining traditional price-based policies with policies based on order book information is investigated. Order books ensure that a trader will never pay more for the stock than the limit price he set. Tested policies are Static Order Book Imbalance (SOBI), Volume Average Weighted Prices (VWAP), Trend Following (TF) and Reverse Policy. SOBI buys (sells) if order book sell prices are greater (smaller) than the order book buy prices. VWAP buys (sells) if the markets average buying (selling) prices are greater (smaller) than VWAP buying (selling) prices. TF calculates a long and a short trend line from 
ticker prices and buys (sells) if slopes of long (short) and short (long) match (both negative / positive). The fourth policy implemented is the reverse strategy discussed in [FRY04]. All four policies were tested over a 15-day period from 2004.01.05 to 2004.01.23 with NASDAQ order book information. Three mixed policies which combine 2, 3 or all of the four policies were considered: SOBI + VWAP + Reverse + TF, SOBI + Reverse and SOBI + Reverse + TF. Results compare returns and Sharpe ratio. For a period length of 15 days the best combined policy is SOBI + Reverse + TF in terms of return, the reverse policy is the overall winner in terms of Sharpe ratio.

\section{Experimental Results}

We want to investigate the performance of the trading policies discussed in Section 3 using experimental analysis. Tests are run for all $p=1,2, \ldots, 6$ period types with the number of periods $n(p)$ from $\{52,26,13,4,2,1\}$ and period length $h$ from $\{7,14,28,91,182,364\}$ days. The following assumptions apply for all tested policies:

1. There is an initial portfolio value greater zero.

2. Buying and selling prices $p_{j}(t)$ of an asset $j$ are the closing prices of day $t$.

3. At each point of time all money is invested either in assets or in $3 \%$ overnight deposit.

4. Transaction costs are $0.0048 \%$ of the market value but between 0.60 and 18.00 Euro.

5. When selling and buying is on different days the money is invested in overnight deposit.

6. At each point of time $t$ there is at most one asset in the portfolio.

7. In each period $i$ at least one buying and one selling transaction must be executed. At the latest on the last but one day of each period asset $j$ has to be bought and on the last day it has to be sold.

8. In period $i=1$ all policies buy the same asset $j$ on the same day $t$ at the same price $p_{j}(t)$; the asset chosen is the one MT will chose (MTasset).

9. In periods $i=2, \ldots, n(p)-1$ trades are carried out according to the different policies.

10. In the last period $i=n(p)$ the asset has to be sold at the last day of that period. No further transactions are carried out from there on.

11. If the reservation price is calculated over $h$ days, the period length is (also) $h$ days.

We simulate all policies using historical XETRA DAX data from the interval 2007.01.01 until 2007.12.31. This interval we divide into $n(p)$ periods where $n(p)$ is from $\{52$, 
$26,13,4,2,1\}$ and $p$ is from $\{7,14,28,91,182,364\}$. With this arrangement we get 52 periods of length 7 days, 26 periods of length 14 days, etc.

We carried out simulation runs in order to find out

(1) if MT shows a superior behaviour to buy-and-hold policies

(2) the influence of $m$ and $M$ on the performance of MT

(3) the average competitive ratio for policies for MA and MT

Two types of buy-and-hold policies are used for simulation; one holds the MTasset within each period $\left(\mathrm{MT} \_\mathrm{B}+\mathrm{H}\right)$ and the other holds the index over all periods (Index_B+H) of a simulation run. Thus, MT_B+H is synchronized with the MT policy, i.e, MT_B+H buys on the first day of each period the same asset which MT buys first in this period (possibly not on the first day) and sells this asset on the last day (note that this asset may differ from the one MT is selling on the last day) of the period. Using this setting we compare both policies related to the same period. Index_B+H is a common policy applied by ETF investment funds and it is also often used as a benchmark although it is not synchronized with the MT policy. In addition to these policies also the random policy Rand is simulated. Rand buys the same asset which MT buys on a randomly chosen day within a holding period.

We first concentrate on question (1) if MT shows a superior behaviour to the policies MT_B+H and Index_B+H. For calculating the reservation prices we use estimates from the past, i.e. in case of a period length of $h$ days $m$ and $M$ are taken from the prices of these $h$ days which are preceding the actual day $t^{*}$ of the reservation price calculation, i.e. $m=\min \{p(t) \mid t$ $\left.=t^{*}-1, t^{*}-2, \ldots, t^{*} h\right\}$ and $M=\max \left\{p(t) \mid t=t^{*}-1, t_{-}^{*} 2, \ldots, t_{-}^{*} h\right\}$. In Table 5-1 the trading results are displayed considering also transaction costs. The return rates are calculated covering a time horizon of one year. For the three active policies (MA, MT, Rand) the transaction costs are the same because all follow the holding period of MT; in all these cases there is a flat minimum transaction fee. 


\begin{tabular}{|l|l|l|l|l|l|l|}
\hline Historic & \multicolumn{6}{|l|}{ Annualized Returns Including Transaction Costs } \\
\hline Policy & $\begin{array}{l}1 \\
n(7)=52\end{array}$ & $\begin{array}{l}2 \text { Weeks } \\
n(14)=26\end{array}$ & $\begin{array}{l}4 \text { Weeks } \\
n(28)=13\end{array}$ & $\begin{array}{l}3 \text { Months } \\
n(91)=4\end{array}$ & $\begin{array}{l}6 \text { Months } \\
n(182)=2\end{array}$ & $\begin{array}{l}12 \text { Months } \\
n(364)=1\end{array}$ \\
\hline MA & $418.18 \%$ & $138.40 \%$ & $201.61 \%$ & $47.93 \%$ & $72.95 \%$ & $61.95 \%$ \\
\hline MT & $41.08 \%$ & $1.37 \%$ & $54.86 \%$ & $6.08 \%$ & $32.39 \%$ & $31.35 \%$ \\
\hline MT_B+H & $9.70 \%$ & $0.50 \%$ & $17.18 \%$ & $15.80 \%$ & $45.30 \%$ & $35.29 \%$ \\
\hline Index_B+H & $20.78 \%$ & $20.78 \%$ & $20.78 \%$ & $20.78 \%$ & $20.78 \%$ & $20.78 \%$ \\
\hline Rand & $-23.59 \%$ & $-21.23 \%$ & $17.18 \%$ & $-18.23 \%$ & $6.20 \%$ & $15.42 \%$ \\
\hline
\end{tabular}

Table 5-1: Annualized Return Rates for different period lengths

MT dominates MT_B+H and Index_B+H in two cases (1 and 4 weeks). MT_B+H dominates MT and Index_B+H in two cases (6 and 12 months). Index_B+H dominates MT and MT_B+H in two cases ( 2 weeks and 3 months). MT generates the best overall annual return rate when applied to 4 weeks. MT_B+H generates the worst overall annual return rate when applied to 2 weeks. MT_B+H policy improves its performance in comparison to Index _B $+\mathrm{H}$ and MT policy proportional to the length of the periods.

We might conclude the longer the period the better the relative performance of MT_B + H. MT outperforms Index B+H in four of six cases and it outperforms MT_B $+\mathrm{H}$ in three of six cases; MT and MT_B+H have the same relative performance. If the period length is not greater than 4 weeks MT outperforms MT_B+H in all cases. If the period length is greater than 4 weeks MT_B+H outperforms $\mathrm{MT}$ in all cases. Index_B+H outperforms MT_B+H in three of six cases.

If we consider the average performance we have $27.86 \%$ for MT, $20.78 \%$ for Index_B+H, and $20.63 \%$ for MT_B+H. MT is not always the best but it is on average the best. From this we conclude that MT shows on average a superior behaviour to buy-and-hold policies under the assumption that $m$ and $M$ are calculated by historical data.

In general we would assume that the better the estimates of $m$ and $M$ the better the performance of MT. Results in Table 5-1 show, that the longer the periods the worse the relative performance of MT. This might be due to the fact that for longer periods historical $m$ and $M$ are worse estimates in comparison to those for shorter periods. In order to analyse the influence of estimates of $m$ and $M$ we run all simulations also with the observed $m$ and $M$ of the actual periods, i.e. we have optimal estimates. Results for optimal estimates are shown in Table 5-2 and have to be considered in comparison to the results for historic estimates shown in Table 5-1. 
Now we can answer question (2) discussing the influence of $m$ and $M$ on the performance of MT. The results are displayed in Table 5-2. It turns out that in all cases the return rate of policy MT improves significantly when estimates of $m$ and $M$ are improved. For all period lengths now MT is always better than MT_B+H and Index_B+H. From this we conclude that the estimates of $m$ and $M$ are obviously of major importance for the performance of the MT policy.

\begin{tabular}{|l|l|l|l|l|l|l|}
\hline Clairvoryant & \multicolumn{6}{|l|}{ Annualized Returns Including Transaction Costs } \\
\hline Policy & 1 Week & 2 Weeks & 4 Weeks & 3 Months \\
& $n(7)=52$ & $n(14)=26$ & Months \\
$n(28)=13$ & $n(91)=4$ & $\begin{array}{l}12 \text { Months } \\
n(182)=2\end{array}$ & $n(364)=1$ \\
\hline MA & $418.18 \%$ & $315.81 \%$ & $280.94 \%$ & $183.43 \%$ & $86.07 \%$ & $70.94 \%$ \\
\hline MT & $102.60 \%$ & $87.90 \%$ & $76.10 \%$ & $81.38 \%$ & $55.11 \%$ & $54.75 \%$ \\
\hline MT_B+H & $9.70 \%$ & $-4.40 \%$ & $22.31 \%$ & $19.79 \%$ & $45.30 \%$ & $35.29 \%$ \\
\hline Index_B+H & $20.78 \%$ & $20.78 \%$ & $20.78 \%$ & $20.78 \%$ & $20.78 \%$ & $20.78 \%$ \\
\hline Rand & $-23.59 \%$ & $-101.3 \%$ & $-10.67 \%$ & $47.37 \%$ & $46.08 \%$ & $15.42 \%$ \\
\hline
\end{tabular}

Table 5-2: Annualized returns for optimal historic estimates

Now we concentrate on question (3) discussing the average competitive ratio for policies MA and MT. We now compare the experimental competitive ratio $c_{e c}$ to the analytical competitive ratio $c_{w c}$. To do this we have to calculate OPT and ON for the experimental case and the worst case. We base our discussion on the return rate $R=s p / b p$ as the performance measure where $s p$ is the selling price and $b p$ is the buying price. We assume that we have precise forecasts for $m$ and $M$.

A detailed example for the evaluation of the competitive ratio is presented in Table 5-3 considering a period length of 12 months. In this period six trades were executed using reservation prices based on the clairvoyant test set. The analytical results are based on the consideration that MA achieves the best possible return and MT achieves a return of zero. E.g. for the first trade MA achieves a return rate of $14.03 \%$ and MT achieves a return rate of $0 \%$ i.e. MT achieves absolutely $14.03 \%$ less than MA and relatively a multiple of 1.1403 . The experimental result are also based on the consideration that MA achieves the best possible return and MT now achieves the return rate generated during the experiment. E.g. for the first trade MA achieves a return rate of $14.03 \%$ and MT achieves a return rate of $13.22 \%$, i.e. MT achieves absolutely $0.82 \%$ less than MA and relatively a multiple of 1.0072 . 


\begin{tabular}{|c|c|c|c|c|c|c|c|c|c|}
\hline \multicolumn{2}{|c|}{ Clairvoyant } & \multicolumn{4}{|c|}{ Analytical Results } & \multicolumn{4}{|c|}{ Experimental Results } \\
\hline $\begin{array}{l}\text { \# Trades } \\
n(364)=1\end{array}$ & \begin{tabular}{|l} 
Holding \\
Period
\end{tabular} & Buy at & Sell at & $\begin{array}{l}\text { Periodic } \\
\text { Return }\end{array}$ & $\begin{array}{l}\text { Ratio } \\
M A / M T\end{array}$ & Buy at & Sell at & $\begin{array}{l}\text { Periodic } \\
\text { Return }\end{array}$ & $\begin{array}{l}\text { Ratio } \\
M A / M T\end{array}$ \\
\hline $1^{\text {st }}$ trade & $\begin{array}{l}\text { Week } \\
1-14\end{array}$ & & & & 1.1403 & & & & 1,0072 \\
\hline MA & & 37,91 & 43,23 & 1.1403 & & 37,91 & 43,23 & 1,1403 & \\
\hline MT & & $p^{*}$ & $p^{*}$ & 1 & & 37,91 & 42,92 & 1,1322 & \\
\hline $2^{\text {nd }}$ & $\begin{array}{l}\text { Week } \\
14-24\end{array}$ & & & & 1.011 & & & & 1,0069 \\
\hline MA & & 34.25 & 38.15 & 1.011 & & 34,25 & 38,15 & 1,1139 & \\
\hline MT & & $p^{*}$ & $p^{*}$ & 1 & & 34,25 & 37,89 & 1,1063 & \\
\hline $3^{\text {rd }}$ & $\begin{array}{l}\text { Week } \\
24-25\end{array}$ & & & & 1.0111 & & & & 1,0000 \\
\hline MA & & 13.54 & 13.69 & 1.0111 & & 13,54 & 13,69 & 1,0111 & \\
\hline MT & & $p^{*}$ & $p^{*}$ & 1 & & 13,54 & 13,69 & 1,0111 & \\
\hline $4^{\text {th }}$ & $\begin{array}{l}\text { Week } \\
25-30 \\
\end{array}$ & & & & 1.0643 & & & & 1,0167 \\
\hline MA & & 33.57 & 35.73 & 1.0643 & & 33,57 & 35,73 & 1,0643 & \\
\hline MT & & $p^{*}$ & $p^{*}$ & 1 & & 34,13 & 35,73 & 1,0469 & \\
\hline $5^{\text {th }}$ & $\begin{array}{l}\text { Week } \\
30-46\end{array}$ & & & & 1.1489 & & & & 1,0646 \\
\hline MA & & 51.23 & 58.86 & 1.1489 & & 51,23 & 58,86 & 1,1489 & \\
\hline MT & & $p^{*}$ & $p^{*}$ & 1 & & 52,37 & 56,52 & 1,0792 & \\
\hline $6^{\text {th }}$ & $\begin{array}{l}\text { Week } \\
46-52 \\
\end{array}$ & & & & 1.0881 & & & & 1,0061 \\
\hline MA & & 82.16 & 89.4 & 1.0881 & & 82,16 & 89,4 & 1,0881 & \\
\hline MT & & $p^{*}$ & $p^{*}$ & 1 & & 82,66 & 89,4 & 1,0815 & \\
\hline
\end{tabular}

Table 5-3: Periodic results for period length one year

We compared the analytical results with the experimental results based on annualized returns for the period lengths 1, 2, 4 weeks, 3, 6, and 12 months. The overall annual return rates for all period lengths are presented in Table 5-4. Transaction costs are not taken into account in order not to bias results. As the policies are always invested there is no overnight deposit. 


\begin{tabular}{|l|l|l|l|l|l|l|l|l|}
\hline Clairvoyant & \multicolumn{2}{l|}{ Ratio } & \multicolumn{2}{l|}{ Annualized Return } \\
\hline Period & \# Trades & OPT/1 & OPT/ON & $1 /$ ON & MA & MT & MA-MT & MT/MA \\
\hline $\begin{array}{l}12 \text { Month } \\
n(364)=1\end{array}$ & 6 & 1.7108 & 1.1045 & $64.56 \%$ & $71.08 \%$ & $54.89 \%$ & $16.19 \%$ & $77.22 \%$ \\
\hline $\begin{array}{l}6 \text { Months } \\
n(182)=2\end{array}$ & 7 & 1,8624 & 1.1994 & $64.40 \%$ & $86.24 \%$ & $55.28 \%$ & $30.96 \%$ & $64.10 \%$ \\
\hline $\begin{array}{l}3 \text { Months } \\
n(91)=4\end{array}$ & 18 & 2.8387 & 1.5613 & $55.00 \%$ & $183.87 \%$ & $81.82 \%$ & $102.05 \%$ & $44.50 \%$ \\
\hline $\begin{array}{l}4 \text { Weeks } \\
n(28)=13\end{array}$ & 38 & 3.8185 & 2.1571 & $56.49 \%$ & $281.85 \%$ & $77.02 \%$ & $204.83 \%$ & $27.33 \%$ \\
\hline $\begin{array}{l}2 \text { Weeks } \\
n(14)=26\end{array}$ & 48 & 4.1695 & 2.2055 & $52.90 \%$ & $316.95 \%$ & $89.05 \%$ & $227.90 \%$ & $28.10 \%$ \\
\hline $\begin{array}{l}1 \text { Week } \\
n(7)=52\end{array}$ & 52 & 4.1711 & 2.0462 & $49.06 \%$ & $317.11 \%$ & $103.84 \%$ & $213.27 \%$ & $32.75 \%$ \\
\hline Average & & & & $57,07 \%$ & $209.52 \%$ & $76.98 \%$ & $132.53 \%$ & $45.67 \%$ \\
\hline
\end{tabular}

Table 5-4: Competitive ratio and annualized return rates

For the period of 12 months the analytical worst case ratio (OPT/1) is 1.7108 and the average experimental ratio $(\mathrm{OPT} / \mathrm{ON})$ is 1.1045 . The percentage the experimental ratio reaches of the worst case ratio which can be calculated by $(1 / \mathrm{ON})$ is $64.56 \%$. The values for the other period lengths are also given in Table 5-4. It turns out that the average experimental ratio reaches at least $49.06 \%$, at most $64.56 \%$ and on average $57.07 \%$ of the analytical worst case ratio; the longer the period the closer is the experimental ratio to the analytical ratio. In terms of return the experimental return reached by MT reaches at least $27.33 \%$, at most $77.22 \%$ and on average $45.67 \%$ of the analytic and experimental return of MA.

\section{Conclusions}

We carried out several experiments to answer three questions. The first is whether MT shows a superior behaviour to buy-and-hold policies or not. The second discusses the influence of estimates for upper and lower bounds, $m$ and $M$, for asset prices on the performance of MT. The third question asks for the average competitive ratio for policies MA and MT.

In order to answer these questions six clairvoyant simulations with optimal estimates for $m$ and $M$ as well as six simulations with historical estimates for $m$ and $M$ were performed. To answer the first question MT outperforms buy-and-hold in all cases even when transaction costs are incorporated in the clairvoyant test set. Simulations based on historical estimates of $m$ and $M$ show that MT outperforms buy-and-hold in one third of the cases and on average. We conclude that if the period length is small enough MT outperforms $\mathrm{B}+\mathrm{H}$. 
From this we can answer the second question discussing the influence of $m$ and $M$ on the performance of MT. It is obvious that the better the estimates of $m$ and $M$ the better the performance of MT. Results show that the longer the periods, the worse are estimates by historical $m$ and $M$ in comparison to those for shorter periods. As a result, the performance of MT gets worse the longer the periods become.

The third question asks for the average competitive ratio for policies MA and MT. Fortunately, these results show that it is very difficult to get close to the (analytical) worst cases under (simulated) real-life considerations. It turns out that the shorter the periods are the less MT achieves in comparison to MA. The longer the periods, the closer is the experimental ratio to the analytical ratio. A MT trading policy which is applied to short periods leads to small intervals for estimating historical $m$ and $M$. In this case there is a tendency to buy too late (early) in increasing (decreasing) markets and to sell too late (early) in decreasing (increasing) markets due to unknown overall trend directions, e.g. weekly volatility leads to wrong selling decisions during an upward trend.

The paper leaves also some open questions for future research. One is that of better forecasts of future upper and lower bounds of asset prices to improve the performance of MT. The suitable period length for estimating $m$ and $M$ is an important factor to provide an optimal trading signal, e.g. if the period length is $h$ days estimates for historical $m$ and $M$ were also be calculated over $h$ days. Simulations with other period lengths for estimating $m$ and $M$ could be of interest. Moreover, the data set of one year is very small. Future research should consider intervals of 5,10 , and 15 years.

\section{References}

Yan98 El-Yaniv,R., Competitive solutions for online financial problems, ACM Computing Surveys 30 (1), 28-69, 1998

YFKT01 El-Yaniv, R., Fiat,A., Karp,R., Turpin,G., Optimal search and one-way trading algorithm, Algorithmica 30, 101-139, 2001

YFKT92 El-Yaniv,R., Fiat,A., Karp,R., Turpin,G., Competitive analysis of financial games, IEEE Symposium on Foundations of Computer Science 327-333, 1992

She02 Shen,P. Market Timing Policies that Worked, Working Paper RWP 02-01, Research Division, Federal Reserve Bank of Kansas City, May 2002

CE08 Chavarnakul,T., Enke,D., Intelligent technical analysis based equivolume charting for stock trading using neural networks, Expert Systems and Applications 34, 1004-1017, 2008 
RL99 Ratner,M., Leal,R.p.C., Tests of technical trading policies in the emerging equity markets of Latin America and Asia, Journal of Banking and Finance 23, 18871905, 1999

FRY04 Feng,Y., Ronggang,Y., Stone,P., Two Stock Trading Agents: Market Making and Technical Analysis, In: Peyman Faratin, David C. Parkes, Juan A. Rodriguez-Aguilar and William E. Walsh (eds.), Agent Mediated Electronic Commerce V: Designing Mechanisms and Systems, Lecture Notes in Artificial Intelligence, 18-36, Springer Verlag, 2004

RS03 Ronggang,Y., Stone,P., Performance Analysis of a Counter-intuitive Automated Stock Trading Stategy, ACM International Conference Proceeding Series Vol. 50, In: Proceedings of the 5th International Conference on Electronic Commerce, Pittsburgh, Pennsylvania, 40-46, 2003

SR05 Silaghi,G.C., Robu,V., An Agent Policy for Automated Stock Market Trading Combining Price and Order Book Information, ICSC Congress on Computational Intelligence Methods and Applications, 4-7, 2005 